\title{
Pragmatic Acquisition and Teaching Chinese as a Foreign Language
}

\author{
Kaijie Chen
}

\author{
Northwest University, Xian 710069, China
}

Keyword: Pragmatic acquisition; Teaching chinese as a foreign language; Improvement measures

\begin{abstract}
From the basic characteristics, teaching Chinese as a foreign language is a key discipline in the linguistic system, which involves the basic linguistic principles of foreign language and the specific context which it is closely related to. Therefore, it can be seen that learning Chinese as a foreign language helps to enhance students' oral and external communication skills. In recent years, new curriculum reform has been fully promoted. In the face of the new situation, the teaching mode of teaching Chinese as a foreign language will also be subject to corresponding changes. In essence, the key to learn Chinese as a foreign language should be pragmatic acquisition. It is necessary to have a comprehensive grasp of a variety of pragmatic habits. Therefore, for the current Chinese class, pragmatic acquisition should be placed in the core position, combined with the real classroom situation of Chinese as a foreign language, to explore the improvement measures.

In many cases, even if there is a relatively strong language ability, the student's corresponding pragmatic competence is not able to obtain synchronic improvement. This is because language communication itself has prominent systematical characteristics, which should contain specific context, communication motivations and other related elements. In the perspective of pragmatic acquisition, students should be able to fully understand and output the related language construction information when facing specific statements [1]. As a result, pragmatic acquisition should be taken as fundamental support during the whole process of learning Chinese. Teachers and students have to be concerned with the pragmatic acquisition of foreign language, in order to from the source optimize their own learning experience of foreign language and then to closely combine with the pragmatics and Chinese language learning.
\end{abstract}

\section{The Basic Principle of Pragmatic Acquisition}

In essence, pragmatic acquisition should be included in the structure of linguistics, and pragmatics itself also constitutes a key branch of the discipline. Thus, pragmatic acquisition refers to learning specific language habits, and then using them in a specific context, to obtain the corresponding pragmatic content [2]. In other words, the fundamental feature of pragmatic acquisition is to construct a specific language context, to use a language by contextual expression and to give a deeper understanding of this. As a result, pragmatic acquisition should fundamentally belong to Context. The meanings of a certain language both explicit and implicit should be given a deep interpretation, based on which, build a diverse contextual relationship.

In recent years, teaching Chinese as a foreign language has received more attention, which is rooted in the marketization and the fierce competition of internationalization. In the classroom of Chinese as a foreign language, Chinese is regarded as a foreign language or a second language. Therefore, fundamentally speaking, Chinese as a foreign language should be classified as a specific category of language teaching. Therefore, pragmatics should be paid more attention [3]. This is because in the face of Chinese specific context, only by learning the relevant Chinese grammar, can students successfully enter a specific context. If teaching Chinese as a foreign language is not based on pragmatic acquisition which provides a fundamental support, then there will be a lack of specific contextures. 


\section{The Specific Ways of Combining Teaching Chinese as a Foreign Language with Pragmatic Acquisition}

Students should consider pragmatic acquisition as a key and primitive factor in learning Chinese as a foreign language. This is because whether pragmatic acquisition has a prominent effectiveness or not directly determines the overall language learning effectiveness. On the contrary, it will have no value to consider pragmatic without the specific communication environment. In other words, the language fragment should not be isolated, but to be integrated into the overall scope of pragmatics, in order to explore the specific types of language habits and other elements. From the current situation, teaching Chinese as a foreign language has been more and more concerned by teachers and students [4]. If students want to improve their own overall level in using Chinese to complete the daily communication, then it is necessary to focus on the most basic pragmatic acquisition. Specifically in practice, teaching Chinese as a foreign language is also committed to the following changes:

Cooperation between Teachers and Students In the entire classroom of teaching Chinese as a foreign language, the full exchange between teachers and students is necessary. Teaching Chinese as a foreign language has to closely integrate with the daily language communication, in order to highlight its true value. Therefore, in the entire course, teachers and students should be committed to cooperate with each other and to strengthen real-time classroom communication. In accordance with the relevant principles of pragmatic acquisition, students who want to improve their existing language using level need to pay special attention to the specific context of the exchange of each other.

So in the current classroom, teachers need to encourage students to try to apply their own existing pragmatic principles to the entire classroom. In recent years, the group collaboration model is being introduced into the daily teaching of Chinese as a foreign language [5]. Within the study group, students should be committed to working closely with each other, to explore key disciplinary knowledge points in teaching Chinese as a foreign language and to ensure that they have a higher level of language application value.

Closely Integrated Context. A specific context should be taken as the background in Chinese language classroom. Teachers and students together create a specific context in order to form a bridge between each other. At the same time, the key to pragmatic acquisition should also be implemented in contextual construction. In the traditional classroom model, college teaching is often inclined to focus on the most basic language habits and other outline knowledge points. In essence, however, the above model is likely to be out of a particular context, so that the entire classroom cannot be integrated into the existing cultural context. Thus, language learning should be closely integrated with the context of the whole language, and teachers and students cannot ignore the comprehensive use of language background.

There is an intrinsic link between the principle of pragmatic acquisition and the daily inter-culture communication. Therefore, in the classroom, teachers need to use a variety of ways to cultivate students to have excellent language habits. From the current situation, the multimedia is fully integrated into classroom. Therefore, when teaching key knowledge points, teachers can use multimedia to show interesting and intuitive pragmatic background for students [6]. On the basis of creating a specific language background, the overall level of oral communication of students can be improved in all directions

Comply with Specific Pragmatic Principles. The Chinese language itself is related to the daily inter-culture communication. In the above process, it is necessary for students to follow the most basic rules. Only in accordance with the rules of pragmatic principles can teachers and students be closely combined with the language context and the context of the special background, and can learn the daily language habits indirectly or directly [7]. In general, the basic principles involved in pragmatic acquisition should include principles of cooperation, politeness and other principles. Specifically in the application of different types of Chinese statements, the above principles should be fully applied.

For example, in the principle of politeness, in general, it will be related to the principle of proper 
language, the principle of praise, generosity and so on. In a variety of language background, teachers and students should pay more attention to the principle of courtesy and decent principles. In other words, when using Chinese to complete a variety of daily oral communication, students should uphold the most fundamental principles of politeness [8]. The use of more appropriate words will make the participators in the oral communication be comfortable and not embarrassing. In addition, the principle of praise should be used moderately and more respect for the parties should be given. Praise words will make the other party have a good feeling of being respected. Therefore, in communication, commendatory words need to be more used.

\section{Conclusion}

After a comprehensive analysis, we can see that teaching Chinese as a foreign language cannot be divorced from Pragmatics and pragmatic acquisition should be integrated into the whole process of classroom teaching. From the current situation, the pragmatic acquisition has been more concerned by teachers and students, they are also working to improve the current stage of the classroom model [9]. In essence, pragmatic acquisition contains a number of levels, among which the key are language and culture theory, context theory, pragmatic principles and other related theories. In the future practice, teachers and students also need to constantly explore, to be committed to optimizing the existing classroom course of teaching Chinese as a foreign language and to optimize the overall classroom experience in the perspective of pragmatic acquisition.

\section{Reference}

[1] Y. Zhang. Survey and Research on Overseas Students' Chinese Pragmatic Competence Acquisition -With overseas students from Ningxia University as example. International conference on economic management and social science: ICEMSS, 2012,12(1):74-75.

[2] Y Nibun, G Wigglesworth. Early pragmatic differentiation in Japanese and German: a case study of a developing trilingual child in Australia. International journal of multilingualism, 2014, 11(1):76-96.

[3] N Katsos, D V M Bishop. Pragmatic tolerance: Implications for the acquisition of informativeness and implicature. Cognition: International Journal of Cognitive Psychology, 2011, 120(1):67-81.

[4] T T Minh Nguyen. Instructional effects on the acquisition of modifiers in constructive criticism by EFL learners. Language awareness, 2013,22(1):76-94.

[5] J Gracer, B Barclay, A Schifrin et al.Pragmatic Environmental Diligence Needed to Stay Competitive in Acquisition Race. Natural Gas and Electricity.,2017,23(9):9-13.

[6] P A Levin, Q Zhang, J H Mersey et al. Glycemic control with insulin glargine plus insulin glulisine versus premixed insulin analogues in real-world practices: a cost-effectiveness study with a randomized pragmatic trial design. Clinical therapeutics, 2011, 33(7):841-850.

[7] N Kazuhiko. Non-insertional code-switching in English-Japanese bilingual children: alternation and congruent lexicalization. International journal of bilingual education and bilingualism, 2012, 15(4):455-473.

[8] Andrés, C Adrian, J E, R A Clemente, et al. Which are the best predictors of theory of mind delay in children with specific language impairment?. International journal of language \&amp; communication disorders, 2013, 48(6):726-737.

[9] P Kim, R Pedone, M Villanti et al. Robust frame synchronization for the DVB-S2 system with large frequency offsets. International journal of satellite communications and networking, 2012, 27(1):35-52. 